\title{
Applying Energy-Equal Entropy of Wavelet Packet to Diagnose Circuit Breaker Faults
}

\author{
Liu Mingliang ${ }^{1,2}$, Wang Keqi ${ }^{1{ }^{1 *}}$, Sun Laijun ${ }^{2}$ and Zhang Jianfeng ${ }^{2}$ \\ ${ }^{1}$ College of Mechanical and Electrical Engineering, Northeast Forestry University, Harbin, China, 150040 \\ ${ }^{2}$ HLJ Province Key Lab of Senior-Education for Electronic Engineering (Heilongjiang University), Harbin, China, \\ 150080
}

\begin{abstract}
Aiming to better reflect features of machinery vibration signals of high-voltage (HV) circuit breaker (CB), a new method is proposed on the basis of energy-equal entropy of wavelet packet(WP). First of all, three-layer wavelet packet decomposes vibration signal, reconstructing 8 nodes of signals in the 3rd layer. Then, the vector is extracted with energy-equal entropy of reconstructed signals. At last, the simple back-propagation (BP) neural network for fault diagnosis contributes to classification of the characteristic parameter. This technology is the basis of a number of patents and patents pending, which is experimentally demonstrated by the significant improvement of diagnose faults.
\end{abstract}

Keywords: Characteristic entropy, energy-equal, fault diagnosis, wavelet packet.

\section{INTRODUCTION}

WP algorithm provides a more precise technique for signal frequency band, thus effectively improving the frequency resolution. Wavelet packet decomposition (WPD) is very effective in decomposing signals. WP achieves a range of frequency bands on the consistent selectivity through splitting signals; at the same time, WP energy presents heterogeneous nature in the different frequency signals $[1,2]$. Stress concentration causes the strength of the signal, and WP band energy can show such power, thus, the feature of crack defect signals can be figured out effectively [3, 4].

On account of statistics and probability, information entropy can describe the uncertainty of the systems [5]. Recently, it was widespread applied in segmentation of data, biomedical, fault diagnosis, signal extraction, and so on; a number of preliminary consequences were obtained $[6,7]$.

HV CB controls and protects power system's operation; hence the running status of CB can directly affect the stability of power network. There is a ton of research on power system shows that $80 \%$ of the HV CB faults are mechanical fault $[8,9]$. As a result, it is of great significance to carry out researches on mechanical fault. The operation of HV CB can produce mechanical vibration signals that contain a large amount of information, reflecting circuit breaker status. The screw loosening and the contactor wearing, etc, can be detected through analyzing the vibration signals $[10,11]$. In past years, research on extracting characteristic parameters out from circuit breaker vibration signals has become very popular. For example, Hu Xiaoguang and his team adopted 3-layer WPD to extract the energy entropy, then to calculate Euclidean distance as the parameters of fault diagnosis [10]. Rong Mingzhe and his team combined the WP extraction algorithm with the short time analysis to process machinery signals, and to analyze the switching synchronization of high circuit breakers [12]. Shen $\mathrm{Li}$ and his team adopted multilayer WP envelope maximum extracting singularity index, then singularity index were used as the feature parameters to reflect the condition of circuit breaker [13]. The authors had applied wavelet packet decomposing vibration signals, and then extracting sensitive nodes for faults, then sensitive nodes were regarded as the feature nodes to reconstruct the signal for fault diagnosis [14]. The above experiment from experts has obtained the good results.

A new method is proposed on the basis of energy-equal entropy of WP. First of all, vibration signals of CB were getting rid of the noise, then, the three-layer wavelet packet decompose vibration signal, reconstructing eight signals of each nodes at the third layer. We put that standard signal energy time segment and compute the integral energy entropy. At last, the simple back-propagation (BP) neural network for fault diagnosis contributes to classification of the characteristic parameter. No-load cases, several faults of high voltage circuit breaker experiment were done.

\section{WAVELET PACKETS}

Based on orthogonal wavelet, the advantages of wavelet analysis are which has good time-frequency localization properties, and has the unique superiority for time-varying signal processing. However, the disadvantage of wavelet analysis is that high time resolution whereas low frequency 


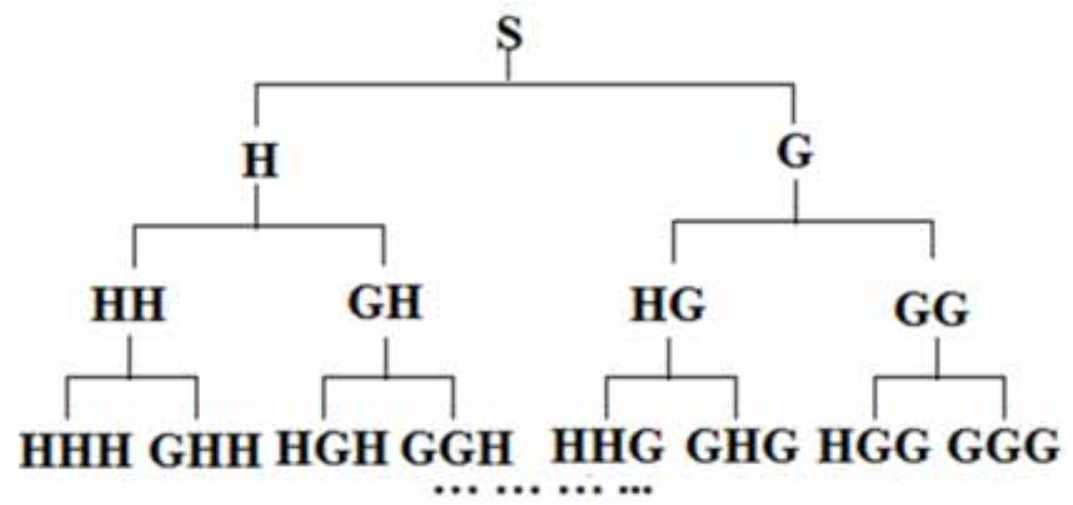

Fig. (1). WPD.

Table 1. Wavelet decomposition frequency range.

\begin{tabular}{|c|c|c|c|c|c|}
\hline Node & $\mathbf{W}(\mathbf{n}, \mathbf{0})$ & $\mathbf{W}(\mathbf{n}, \mathbf{1})$ & $\mathbf{W}(\mathbf{n}, \mathbf{2})$ & $\mathbf{W}(\mathbf{n}, \mathbf{3})$ & $\ldots \ldots$ \\
\hline \hline$f_{m}$ & $0 \sim 1 / 2^{\mathrm{n}}$ & $1 / 2^{\mathrm{n}} \sim 2 / 2^{\mathrm{n}}$ & $3 / 2^{\mathrm{n}} \sim 4 / 2^{\mathrm{n}}$ & $2 / 2^{\mathrm{n}} \sim 3 / 2^{\mathrm{n}}$ & $\ldots \ldots$ \\
\hline
\end{tabular}

resolution in high part of time-frequency resolution. Nonetheless, WP overcomes that disadvantage. Applying of wavelet packet decomposition, fault diagnosis effect of $\mathrm{HV}$ CB was very good $[15,16]$.

Provided conjugate filter $k_{n}=k(n)$ :

$\sum k(n-2 l) k(n-2 m)=\delta_{l m}$

$f_{n}=f(n)=(-1)^{n} k_{1-n}$

Based on the double scales, a group of recursive functions $\left\{p_{n}(x)\right\}_{n \in z}$ is defined as:

$\left\{\begin{array}{l}p_{2 n}(x)=\sqrt{2} \sum k_{l} p_{n}(2 x-l) \\ p_{2 n+1}(x)=\sqrt{2} \sum f_{l} p_{n}(2 x-l)\end{array}\right.$

$f_{l}, k_{l}$ are low-pass filter and high-pass filter groups respectively.

WPD successfully keeps frequency-time localized features of signals. Within the full frequency, in each layer wavelet-packet decomposes sub-band into two parts, then repeating the appeal in the each part. Following that, a form of binary tree is constructed, which is shown as Fig. (1). Wavelet decomposition frequency range was shown as Table 1. Covering the full frequency of signals, resolutions of each layer is different $[16,17]$.

\section{ENTROPY}

The homogeneity of the energy distribution of system can be described by information entropy [8,18-20].

Provided an system with $\mathrm{M}$ random states $x_{1}, x_{2} \ldots x_{M}$, corresponding probability are $p_{1}, p_{2}, \ldots, p_{M}$, So corresponding information entropy can be defined as:
$H=-\sum_{i=1}^{M} p_{i} \log p_{i}$

Fault diagnosis of circuit breaker is essential for normal state and fault state to distinguish. The fault can be seen as various mutations in the Reliable state. According to this property, we can use the piecewise approach to achieve entropy extraction.

This paper presents a scheme for diagnose circuit-breaker mechanical fault based on energy-equal entropy. The specific operation is in accordance with the integral energy equivalent principle, the standard envelope signal time axis segmentation, and the segmentation method is applied to segment the test signal.

In Fig. (2), Signal1 as normal signal envelope, Signal2 as the fault signal envelope. Mutation event were delayed, Sig2 compared with Sig1. Signal1 was segmented according to energy-equal; each of them has three segments Seg1, Seg2, Seg3. Because the Signal2 relative to the Signal1 state is changed, so the Signal2 Seg1, Seg2, Seg3 energy relative to the Signal1 Seg1, Seg2, Seg3 energy has also changed, namely energy distribution changes. So we will change Signal1, Signal2 corresponding to each segment of the transformation of the change of energy distribution uniformity.

\section{METHODOLOGIES}

The procedure to diagnose Wavelet Packet- characteristic entropy fault is as follows [5, 14, 16, 21]:

Step 1: After soft-threshold de-noising, state signals are respectively wavelet packet decomposed at the $n$ layers. After many times of test, selecting db10 as base wavelet to reconstruct signals in $2 \mathrm{n}$ nodes at the $\mathrm{n}$ layer;

Step 2: Respectively figure out the envelope energy of reconstituted signals shown in Step1 with Hilbert transform. 


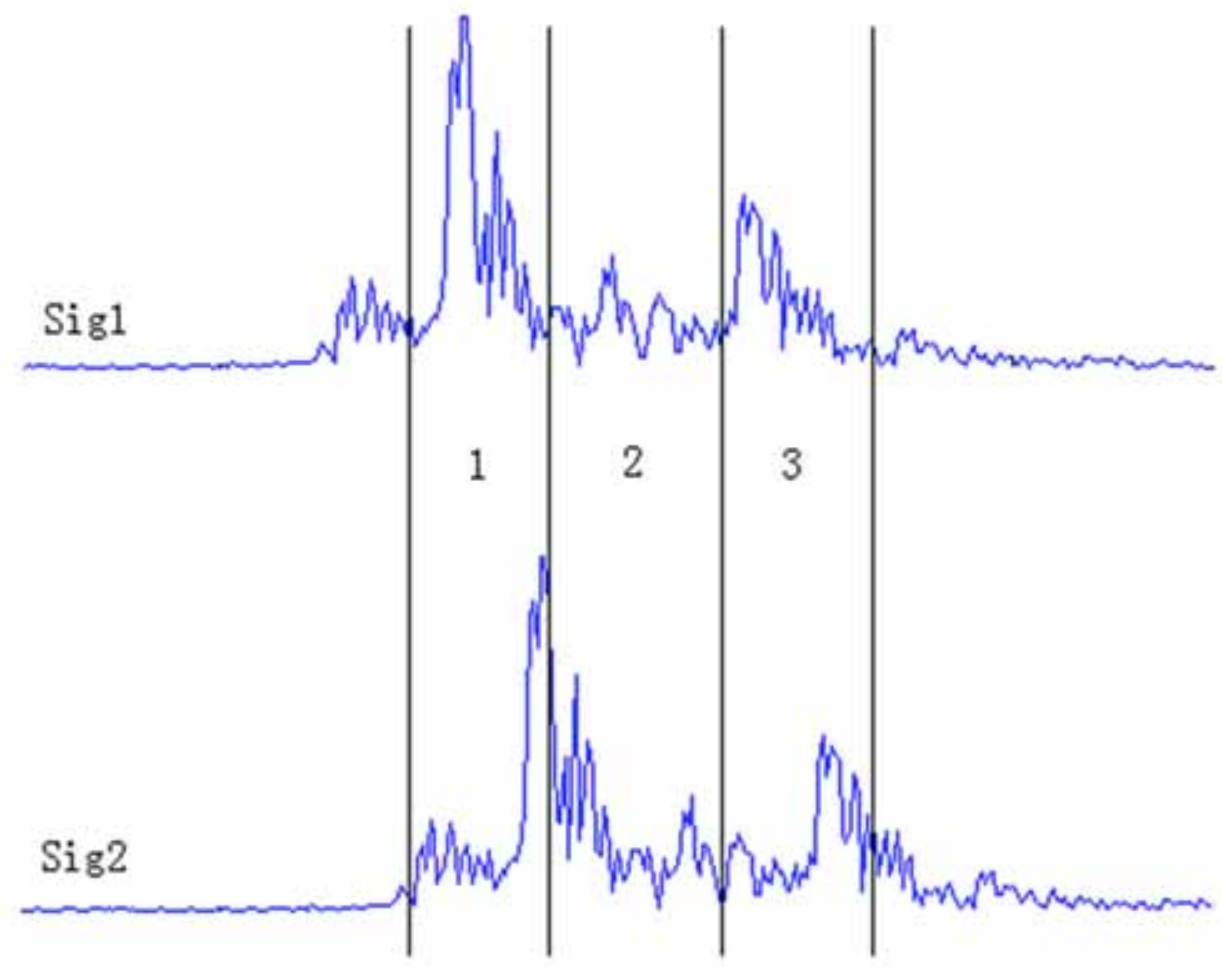

Fig. (2). Segments with equal energy.

Step 3: The envelope signal of each node was divided into $M$ parts in accordance with the principle of equal energy along the time axis, the piecewise point in time was extracted.

Step 4: A vibration signal, by step1 and step2, the envelope signal was divided into $\mathrm{M}$ parts according to the obtained piecewise points of normal signal, and the use of Equation (3) to energy.

$Q_{l}(i)=\int_{t_{1}}^{t_{2}}|f(x)|^{2} d x$

In the formula, $i=1,2, \cdots, m, \quad l=1,2, \cdots, n \quad t_{1}, t_{2}$ the start and stop time.

Step 5: The energy was normalized as Equation (4):

$\varepsilon_{l}(i)=\frac{Q_{l}(i)}{\sum_{i=1}^{m} Q_{l}(i)}$

Define characteristics entropy of the signal $x(t)$ is $[14$, 22]:

$H_{l}=-\sum_{i=1}^{m} \varepsilon_{l}(i) \lg \varepsilon_{l}(i)$

Step 6: Wavelet Packet - characteristic entropy vector are:

$T=\left[H_{0}, H_{1}, H_{2} \ldots \ldots H_{2^{n}-1}\right]$
The partition number " $\mathrm{m}$ " is related to complexity of signal, if the vibration events are more in the vibration signal extraction, $\mathrm{m}$ value is large, whereas the $\mathrm{m}$ value is small. The $\mathrm{n}$ value distribution range size reflects the character frequency, which is the size of the signal frequency resolution.

When we use the method of Wavelet Packet - entropy for fault detection, in fact is the normal state signal as uniform, and in the testing signal under fault condition and its distribution is not uniform, the entropy is a measure of signal heterogeneity degree, so we can use the Wavelet Packet entropy, which can reflect the fault state with respect to the degree of deviation from the normal state [22].

\section{RESEARCH DESIGN}

\subsection{Normal Signal Processing}

Fig. (3) is a vacuum circuit breaker A normal standard signal, and then the signal was processed according to the Wavelet Packet - entropy. We refer to other circuit breaker vibration signal processing method [20]. $\mathrm{n}=3, \mathrm{~m}=15$, A total of 8 node signal reconstruction. The energy fragments of 15 equal time scale were shown in Table 2 . The following shows the analysis of the method by simulating the timevarying signal.

\subsection{Time-variations}

The normal signal in Fig. (3), analogs 5 signal by increasing random noise and delay, which is shown Fig. (4). 
Table 2. Equal energy time point (Unit: $\mathrm{ms}$ ).

\begin{tabular}{|c|c|c|c|c|c|c|c|c|}
\hline & {$[3,0]$} & {$[3,1]$} & {$[3,2]$} & {$[3,3]$} & {$[3,4]$} & {$[3,5]$} & {$[3,6]$} & {$[3,7]$} \\
\hline 1 & 29.19 & 32.00 & 40.16 & 37.01 & 33.83 & 32.04 & 40.96 & 31.50 \\
\hline 2 & 42.37 & 47.03 & 95.29 & 89.93 & 47.01 & 47.89 & 98.19 & 45.21 \\
\hline 3 & 57.51 & 62.71 & 112.80 & 115.51 & 66.97 & 70.36 & 129.61 & 81.70 \\
\hline 4 & 73.39 & 86.05 & 132.51 & 131.91 & 120.96 & 127.81 & 142.79 & 129.60 \\
\hline 5 & 94.01 & 108.81 & 144.70 & 143.41 & 148.81 & 146.89 & 148.74 & 143.31 \\
\hline 6 & 127.03 & 127.35 & 153.60 & 152.39 & 161.49 & 159.99 & 154.93 & 151.97 \\
\hline 7 & 152.42 & 145.09 & 161.59 & 160.62 & 169.61 & 173.90 & 162.60 & 159.19 \\
\hline 8 & 163.41 & 165.21 & 169.69 & 166.49 & 188.91 & 188.38 & 168.13 & 165.20 \\
\hline 9 & 175.99 & 178.04 & 180.70 & 176.92 & 193.31 & 192.39 & 173.27 & 178.03 \\
\hline 10 & 196.22 & 192.46 & 191.59 & 191.20 & 199.82 & 197.49 & 182.99 & 191.10 \\
\hline 11 & 209.38 & 197.60 & 199.40 & 198.10 & 206.72 & 204.40 & 193.02 & 199.01 \\
\hline 12 & 231.29 & 210.29 & 209.38 & 210.82 & 218.58 & 218.11 & 204.50 & 211.99 \\
\hline 13 & 264.09 & 228.99 & 228.93 & 229.99 & 238.19 & 240.87 & 223.20 & 232.21 \\
\hline 14 & 301.60 & 268.30 & 260.48 & 269.21 & 281.06 & 283.29 & 257.29 & 275.57 \\
\hline 15 & 333.49 & 333.46 & 333.49 & 333.49 & 333.49 & 333.49 & 333.46 & 333.49 \\
\hline
\end{tabular}

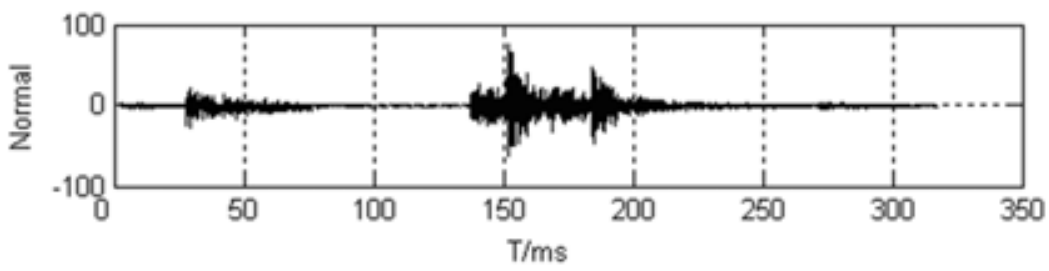

Fig. (3). Standard signal of normal state.

Table 3. Testing entropy vectors of time-variation.

\begin{tabular}{|c|c|c|c|c|c|c|c|c|}
\hline & $\boldsymbol{H}_{\mathbf{0}}$ & $\boldsymbol{H}_{\mathbf{1}}$ & $\boldsymbol{H}_{\mathbf{2}}$ & $\boldsymbol{H}_{\mathbf{3}}$ & $\boldsymbol{H}_{\mathbf{4}}$ & $\boldsymbol{H}_{\mathbf{5}}$ & $\boldsymbol{H}_{\mathbf{6}}$ & $\boldsymbol{H}_{\mathbf{7}}$ \\
\hline \hline$E t 1$ & 1.2500 & 1.1803 & 1.0904 & 1.2248 & 1.2529 & 1.2830 & 1.0697 & 1.1720 \\
\hline$E t 2$ & 1.2359 & 0.9811 & 1.0210 & 1.0258 & 1.1829 & 1.2677 & 0.9630 \\
\hline$E t 3$ & 1.2178 & 0.9503 & 0.9310 & 0.8109 & 1.0701 & 1.2301 & 0.8949 & 0.9180 \\
\hline$E t 4$ & 1.2079 & 0.9068 & 0.8350 & 0.8789 & 1.0089 & 1.1814 & 0.7640 & 0.9737 \\
\hline$E t 5$ & 1.2040 & 0.8810 & 0.6830 & 0.8135 & 1.0049 & 1.1448 & 0.6672 & 0.9180 \\
\hline$E t 6$ & 1.2056 & 0.7878 & 0.7081 & 0.7704 & 0.9535 & 1.0628 & 0.6064 & 0.8026 \\
\hline
\end{tabular}

Et1 is the original vibration signal, Et2 Et6 are the analog signal from $100 \mathrm{~ms} 3 \mathrm{~ms}$ turn delay. Fig. (4) shows that the signal impact event of $100 \mathrm{~ms}$ later turns back from top to bottom. Et6 has been compared to the normal state signal after the shift to $15 \mathrm{~ms}$. According to the characteristic entropy extraction step 4 6, we are processing each signal respectively in Fig. (4), wavelet packet characteristic entropy vector Table 2. Observation of Table $\mathbf{3}$ feature vectors can be 


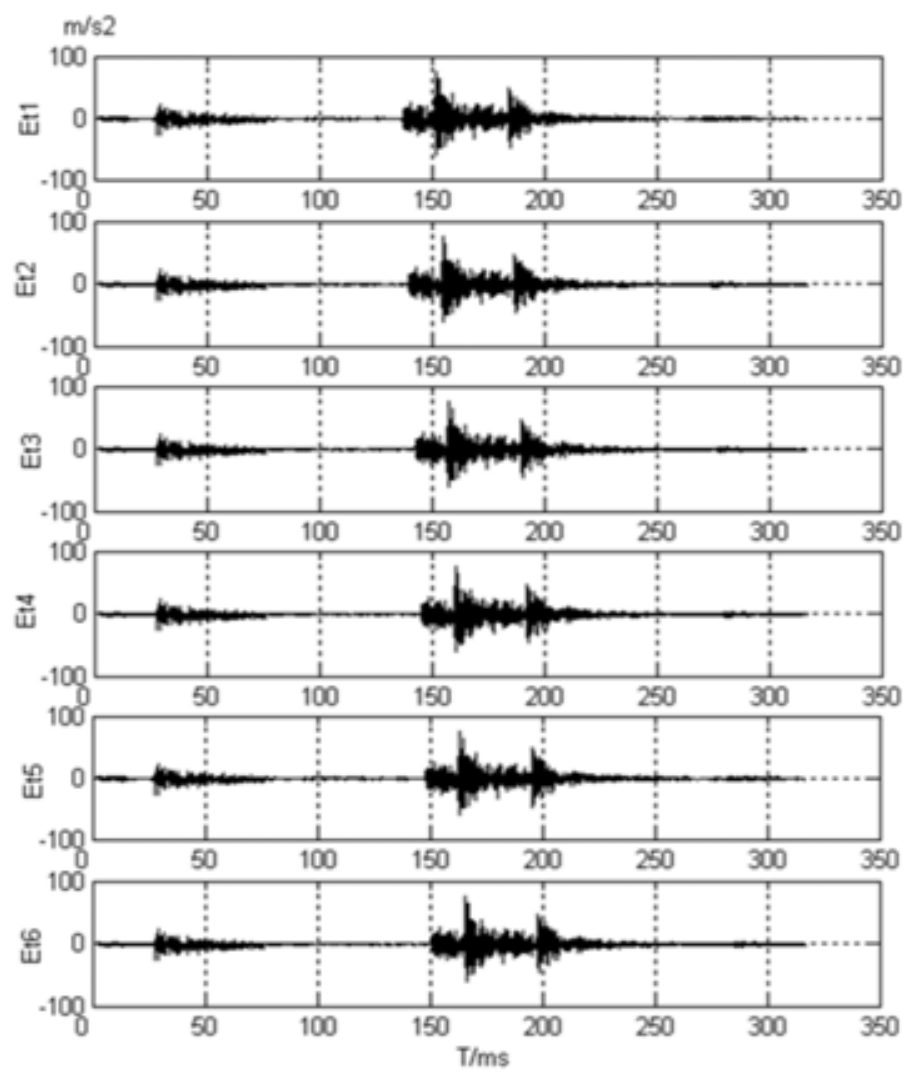

Fig. (4). Simulated signals of time-variation.

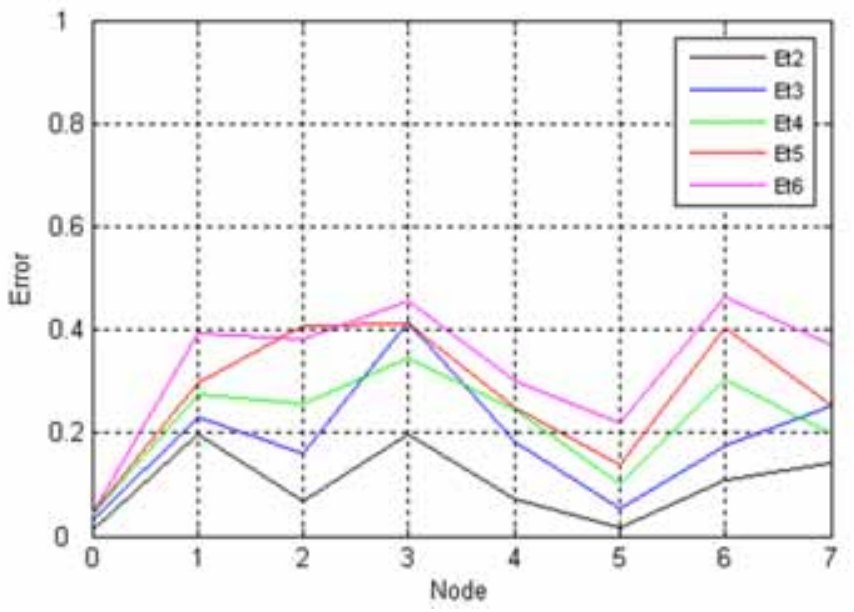

Fig. (5). Variation curves of entropy-variation.

seen, in the normal state vector elements are uniformly distributed, with time delay, the uniform gradually being broken, the entropy decreases. Fig. (5) is variation curves of entropy-variation. The abscissa is node, and the ordinate is the amount of change. With longer time of delay, the nodes were basically the same. Nodes in $[3,0]$ are the lowest frequency. The entropy change is small; this is because compared to the other band, frequency distribution in the signal in the most uniform. Time no matter how to change, will not cause obvious changes in the distribution of low frequency component. Using the formula $\sigma_{i}=\left\|T_{1}-T_{i}^{\prime}\right\|, \quad i=2, \ldots, 6$, we calculate Euclidean distance of the delay signal and the normal signal, as shown in Table 4. As time later, the distance of each signal vector and normal signal increase gradually, each node is consistent.

\subsection{Applications}

Take a vacuum circuit breaker working under no load for test. Firstly, the circuit breaker operation 1 standard sets of vibration signal in the normal state. Then, the poor circuitbreaker lubrication is tried to test time-delay fault. Following that, loose phase A base screw and simulate the condition to 
Table 4. Time-variation Euclidean distance.

\begin{tabular}{|c|c|c|c|c|c|}
\hline & $\left\|T_{1}-T_{2}\right\|$ & $\left\|T_{1}-T_{3}\right\|$ & ||$T_{1}-T_{4} \|$ & $\left\|T_{1}-T_{5}\right\|$ & $\left|T_{1}-T_{6}\right| \mid$ \\
\hline \hline$\sigma$ & 0.8160 & 1.4998 & 1.7664 & 2.2076 & 2.6270 \\
\hline
\end{tabular}
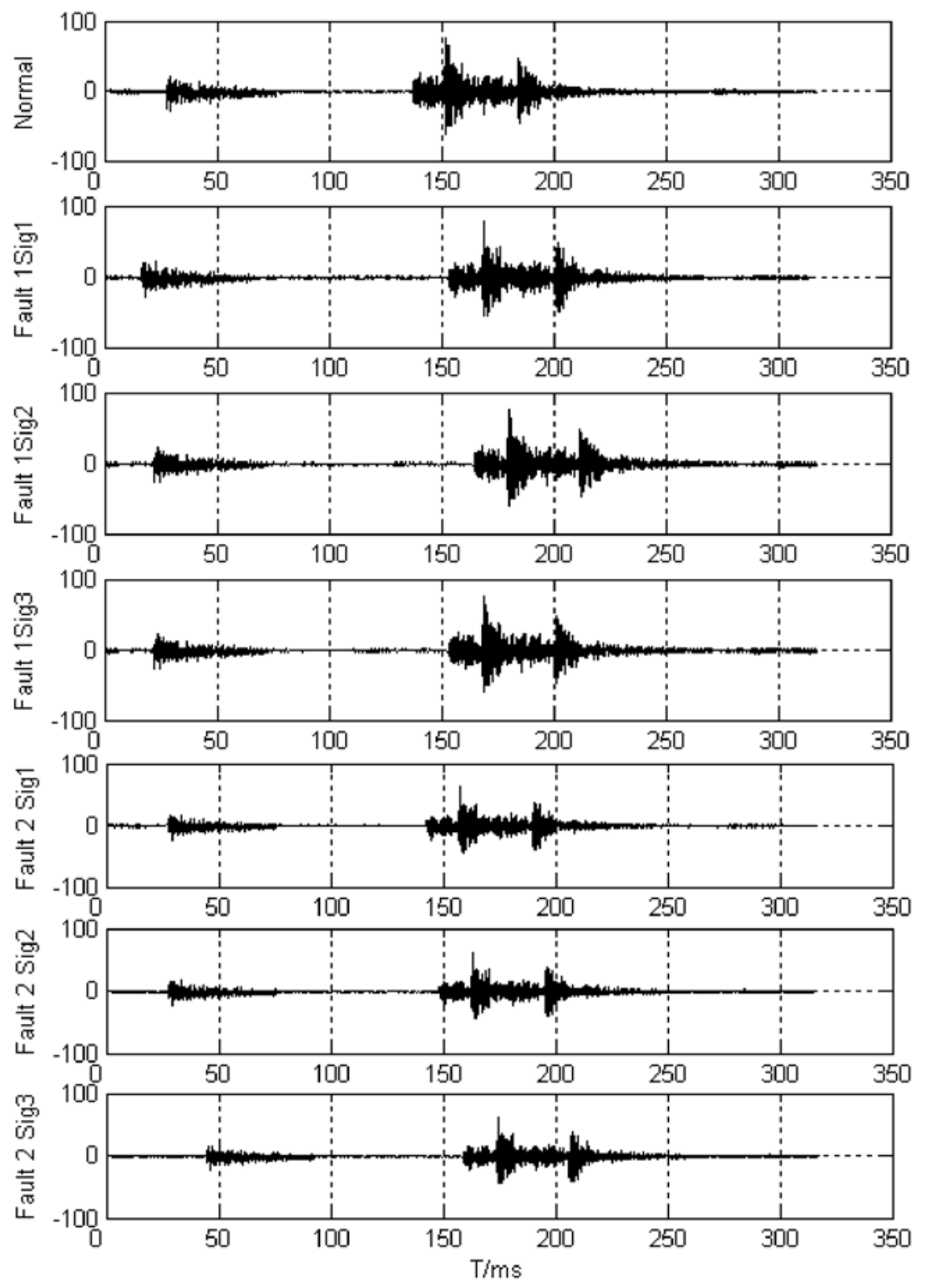

Fig. (6). Original signal of A.

test incorrect fault. Under these 3 conditions circuit breaker operates respectively, continuous action 3 times, 6 sets of data. Finally there are 7 groups of vibration signal as shown in Fig. (6), followed by signal standard signal, lack of lubrication condition ( 3 signal Fault I,), screw loosening signal condition (Fault II, 3 signal) [10, 21].

We process signal in the shown in Fig. (6) according to the 4 of the steps, sub time scale is shown in Table 2 , the signal characteristic entropy vector as shown in Table $\mathbf{5}$. Through the analysis of the vector of table is easily found, The fault signal segmentation using normal condition signals energy distribution model, the original score was broken, so that the entropy change, change with the fault severity. The fault signal entropy vector of each element is generally less than normal, and the elements distribution is scattered, it also shows that in the fault state of each frequency band energy 
Table 5. Vectors of characteristic entropy.

\begin{tabular}{|c|c|c|c|c|c|c|c|c|}
\hline The Type of Fault & $\boldsymbol{H}_{\mathbf{0}}$ & $\boldsymbol{H}_{\mathbf{1}}$ & $\boldsymbol{H}_{\mathbf{2}}$ & $\boldsymbol{H}_{\mathbf{3}}$ & $\boldsymbol{H}_{\mathbf{4}}$ & $\boldsymbol{H}_{\mathbf{5}}$ & $\boldsymbol{H}_{\mathbf{6}}$ & $\boldsymbol{H}_{\mathbf{7}}$ \\
\hline \hline Normal Signal & 1.2495 & 1.1808 & 1.0907 & 1.2257 & 1.2534 & 1.2828 & 1.0698 & 1.1725 \\
\hline Fault I & 1.1321 & 0.8014 & 0.6135 & 0.6345 & 0.8985 & 1.0186 & 0.5068 & 0.7056 \\
\hline Fault I & 1.1892 & 0.7446 & 0.6044 & 0.5392 & 0.8386 & 0.9017 & 0.5208 & 0.6337 \\
\hline Fault I & 1.1888 & 0.7865 & 0.7172 & 0.5687 & 0.9046 & 1.0158 & 0.5425 & 0.6695 \\
\hline Fault II & 1.1644 & 0.8967 & 0.8923 & 0.8352 & 1.0794 & 1.2817 & 0.7966 & 0.8894 \\
\hline Fault II & 1.1275 & 0.8084 & 0.7307 & 0.7655 & 0.9805 & 1.1785 & 0.6417 & 0.8825 \\
\hline Fault II & 1.0882 & 0.6345 & 0.4676 & 0.5494 & 0.7665 & 0.8365 & 0.4076 & 0.6586 \\
\hline
\end{tabular}

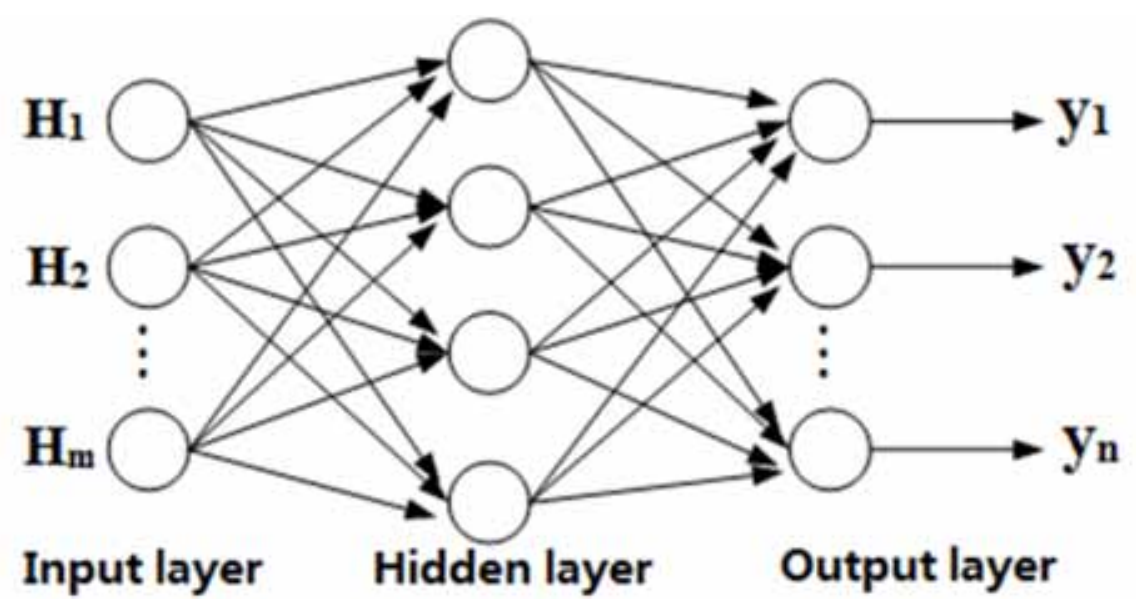

Fig. (7). Structure of neural network.

Table 6. Testing results of neural network.

\begin{tabular}{|c|c|c|c|c|c|c|c|c|c|c|c|c|c|c|}
\hline Out-puts & $\begin{array}{l}\text { Normal } \\
\text { Sig }\end{array}$ & $\operatorname{Exp}$ & $\begin{array}{c}\text { Fault I } \\
\text { Sig } 1\end{array}$ & $\operatorname{Exp}$ & $\begin{array}{c}\text { Fault I } \\
\text { Sig } 2\end{array}$ & $\operatorname{Exp}$ & $\begin{array}{c}\text { Fault I } \\
\text { Sig } 3\end{array}$ & $\operatorname{Exp}$ & $\begin{array}{c}\text { Fault II } \\
\text { Sig } 1\end{array}$ & $\operatorname{Exp}$ & $\begin{array}{c}\text { Fault II } \\
\text { Sig } 2\end{array}$ & $\operatorname{Exp}$ & $\begin{array}{c}\text { Fault II } \\
\text { Sig } 3\end{array}$ & $\operatorname{Exp}$ \\
\hline$y_{2}$ & -0.0277 & 0 & 0.8457 & 1 & 0.8984 & 1 & 0.9258 & 1 & -0.0927 & 0 & -0.0692 & 0 & -0.1157 & 0 \\
\hline$y_{3}$ & 0.0863 & 0 & 0.2766 & 0 & 0.1148 & 0 & 0.0951 & 0 & 0.9600 & 1 & 0.9185 & 1 & 0.9120 & 1 \\
\hline
\end{tabular}

distribution is obviously disturbed, can criterion as a judgment circuit breaker has no fault, At the same time can be found, screw loosening fault signal the entropy is generally less than the time delay fault.

BP neural network was constructed by MATLAB neural network toolbox. Fig. (7) shows its structure. Its input layer possesses 8 neurons corresponding to the 8 nodes entropy. The neural network output layer has 3 neurons corresponding to 3 statuses of circuit breakers. In accordance with training output, the number neurons of hidden-layer were assigned to be 9 , step length was 0.32 , and expected error was
0.005. Network was trained using Table $\mathbf{5}$ vector, the output results as shown in Table 6. The experimental data show better classification results of BP network design. Many experiments prove that feature parameters extracted through this technique can precisely and better discriminate various types of common faults.

In the actual application we can use more data to train the network, but the state data too much can cause that the network training convergence is difficult, even fail to converge. This needs to strike a balance between diagnostic status and the number of diagnostic errors. Therefore, it is essential to 
focus on training fault that often happens in operation causing great harm [23-25].

\section{CURRENT \& FUTURE DEVELOPMENTS}

The paper has reviewed the author's patent on the circuit breaker, and presented an Energy-equal Entropy method on the strength of WP and information entropy. The technique is applied on fault diagnosis and over travels on the circuit breaker itself insufficient lubrication, screw loosening two fault conditions of the method are analyzed, and achieved good simulation effect. At the same time, the simulation results also show that the method can be used for other equipment soft fault diagnosis based on signal processing. However, a lot of samples are needed for improving BP network when applying this patent during the practice.

\section{CONFLICT OF INTEREST}

The authors declare no conflicts of interest.

\section{ACKNOWLEDGEMENTS}

This work is supported by natural science foundation of Heilongjiang province of China (E201233).

\section{REFERENCES}

[1] Wushen Li, Xinjie Di, Shiwu Bai, F. Liu, and Z. Xue, "Feature Analysis of Metal Magnetic Memory Signals for Welding Crack based on Wavelet Energy Spectrum", INSIGHT: Non-Destructive Testing and Condition Monitoring, The British Institute of NonDestructive Testing, Northampton, UK, vol. 48, pp. 426-429, 2006.

[2] F. Yi, Z. Li, Y. Su, "Feature Extraction of Pipeline Crack Defect Signals with MMM Testing Based On Wavelet packet Frequency Bands Energy", In $2^{\text {nd }}$ International Conference on Information Technology and Computer Science, Kiev, 2009, pp.277-280.

[3] Z. Gui, F. Han, "Neural Network Based On Wavelet Packetcharacteristic Entropy for Fault Diagnosis of Draft Tube," In: Proceeding of the CSEE, 2005, vol. 25, no. 4, pp. 99-102

[4] T. Du, G. Chen, Y. Lei, "A novel method for power system harmonic detection based on wavelet transform with aliasing compensation", In: Proceedings of the CSEE 2005, vol. 25, no. 3, pp. 5459.

[5] L. Sun, M. Liu, H. Qian, J. Zhen, "A New Method to Fault Diagnosis for Circuit Breakers Based on Characteristic Entropy of Wavelet Packet", In: $2^{\text {nd }}$ International Conference on Digital Manufacturing \& Automation 2011, pp. 63-67

[6] M. Runde, T. Aurud, L. E. Lundgaard, G. E. Ottesen, K. Faugstad, "Acoustic diagnosis of high voltage circuit-breakers", IEEE Trans. Power Delivery, vol. 7, no. 3, pp. 1306-1315, 1992.
[7] T. H. Probert, "Steganographic Messaging System Using Code Invariants," U. S. Patent 20140108803, April 17, 2014.

[8] M. Liu, L. Sun, J. Zhen, J. Li, "Fault Diagnosis of High Voltage Circuit Breaker Based on multiple entropy strips Method," In 6th IEEE Conf. Indust. Electroni. Appl., Beijing, 2011.498-502.

[9] C. Wang, "Mechanical Parameter on Line Monitoring System for SF6 High Voltage Circuit Breakers", Power Sys. Technol., vol.23, no.7, pp. 46-52, 1999.

[10] Laijun Sun, Xiaoguang Hu, Yanchao Ji, "Fault Diagnosis for HV Circuit Breakers with Characteristic Entropy of Wavelet Packet", Automat. Elect. Power Syst., vol. 26, no. 8, pp.62-65, 2006.

[11] B. Guadalupe, G. Blanco, "Automatic And Self-sustaining Electronic System For The Early Detection Of Short Circuit Fault Conditions". U. S. Patent 20130265681, October 10, 2013.

[12] Q. Ma, M. Rong, S. Jia, "Study of Switching Synchronization of High Voltage Breakers Based on The Wavelet Packets Etractional Gorithm and Short Time Analysis Method", In: Proceeding of the CSEE, 2005, vol. 25, no. 13, pp. 149-154.

[13] L. Shen, Y. Huang, J. Qian, "Research on Machanical Congition Monitoring for HV Circuit Breakers", China Elect. Eng. Proce., 1997, vol.17, no.2, pp. 113-117.

[14] L. Sun, X. Hu, Y. Ji, "A New Method of Fault Diagnosis for High Voltage Circuit Breakers Based on Vibration Signals", In: Proceedings of the CSEE, 2006, vol.26, no.6, pp.157-161.

[15] Z. Cheng, "Wavelet Analysis Algorithms and Application", Xi'an: Xi' an Jiaotong University Press, 1998.

[16] L. Sun, M. Liu, J. Zhen, G. Ye, "A new fault diagnosis method for HV circuit breakers based on wavelet packet-neural network, In: 6th IEEE Conference on Industrial Electronics and applications, Beijing, 2011.844-849.

[17] R. Alaei, S. H. Fathi, and J. Milimonfared, "Harmonic analysis of fault current limiters", In 6th IEEE Conference on Industrial Electronics and applications, Beijing, 2011. pp. 838-843.

[18] R. Rakoczy, M. Kordas, G. Story, M. Konopacki, "The characterization of the residence time distribution in a magnetic mixer by means of the information entropy", Chem. Eng. Sci., vol.105, no.2, 2014, pp. 191-197.

[19] H. Su, J. Hu, Z. Wen, "Structure analysis for concrete-faced rock fill dams based on information entropy theory and finite element method”, Int. J. Num. Anal. Meth. Geomech., 2012, vol. 36, no. 8, pp. 1041-1055.

[20] Y. Li, R. Shao, J. Cao, "A New and Effective Method of Gear Fault Diagnosis Using Wavelet Packet Transform Combined with Support Vector Machine", J. Northwestern Polytech. Univ., 2010, vol. 28 , no. 4 , pp. $530-535$.

[21] L. Su, "Time-variation and Frequency-variation Analysis of Wavelet Packet-characteristic Entropy for Vibration Signals", High Volt. Eng, 2007, vol. 8, no. 8, pp. 146-150.

[22] J. Ma, J. Ye, "Aero-engine Faults Diagnosis Based on Wavelet Packet Analysis. Computer Simulation", 2010 vol. 27, no. 2, pp. 48-52.

[23] K. Saito, H. Kanao "Fault diagnosis apparatus of fuel evaporation/dissipation prevention system”, U. S. Patent 20040068360, April 8, 2004.

[24] R. Iwatsuki, "Storage apparatus and fault diagnosis method", U. S. Patent 20110239090, September 29, 2011.

[25] L. Li, W. Qian, T. D. Lundell "Rotating Machinery Condition Monitoring Using Position Sensor", U. S. Patent 20120126738, May 24, 2012. 